Cahiers Charlevoix

Études franco-ontariennes
Cahiers Charlevoix Études franco-ontariennes

or Crevenerix of

\title{
Le phonème /r/ en franco-ontarien : réalisations et perceptions
}

\section{Ali Reguigui}

Volume 12, 2018

URI : https://id.erudit.org/iderudit/1048918ar

DOI : https://doi.org/10.7202/1048918ar

Aller au sommaire du numéro

\section{Éditeur(s)}

Société Charlevoix

Presses de l'Université d'Ottawa

ISSN

1203-4371 (imprimé)

2371-6878 (numérique)

Découvrir la revue

Citer cet article

Reguigui, A. (2018). Le phonème/r/ en franco-ontarien : réalisations et perceptions. Cahiers Charlevoix, 12, 117-148. https://doi.org/10.7202/1048918ar

\section{Résumé de l'article}

Ali Reguigui inscrit son étude dans le cadre de la phonétique articulatoire et de la sociophonétique, fondée sur les données d'un questionnaire sociolinguistique recueillies auprès de sujets franco-ontariens. Il examine le cas de la consonne roulée alvéolaire voisée / $r$ / du latin, qui a persévéré en ancien français et moyen français jusqu'au $\mathrm{XVII}^{e}$ siècle dans les centres urbains, et jusqu'au $\mathrm{XVIII}^{e}$ siècle en général pour se faire remplacer par la consonne roulée uvulaire voisée, communément qualifiée de grasseyée et marquée comme variante de prestige en France. Au Québec, au début du $\mathrm{xx}^{e}$ siècle, c'est le [r] roulé, associé à l'élite intellectuelle, qui était valorisé. Dévalorisée vers le milieu de ce siècle, cette variante est abandonnée par l'élite au profit de la variante grasseyée et elle marque de plus en plus le langage de la classe populaire. Ces perceptions se traduisent par des jugements de valeur ou des clichés sur l'usage de ces variantes. Qu'en est-il de l'usage et de la perception du phonème / $r$ / chez les Franco-Ontariens, une minorité bilingue qui vit en contact constant avec l'anglais? Quelles sont leurs réalisations du phonème $/ r$ / et quelles en sont leurs perceptions ? Cette étude apporte des réponses à ces questions.
Ce document est protégé par la loi sur le droit d'auteur. L'utilisation des services d'Érudit (y compris la reproduction) est assujettie à sa politique d'utilisation que vous pouvez consulter en ligne.

https://apropos.erudit.org/fr/usagers/politique-dutilisation/ 


\title{
Le phonème $/ \mathbf{r} /$ en franco-ontarien : réalisations et perceptions
}

\author{
Ali Reguigui \\ Département d'études françaises \\ Université Laurentienne
}




\section{SOMMAIRE}

$\begin{array}{ll}\text { INTRODUCTION } & 119\end{array}$

$\begin{array}{lll}\text { I - Contexte } & 121\end{array}$

II - OBJeCtifs de L'ÉtUde 128

Problématique 128

Hypothèses $\quad 129$

Méthodologie 129

III - ANALYSE ET INTERPRÉTATION DES RÉSULtATS 132

Effet du sexe 133

Effet de l'âge 134

Effet du niveau d'instruction 137

Effet des habitudes articulatoires des parents $\quad 139$

Effet de la région où l'on a vécu

le plus longtemps 141

Usage et préférence $\quad 144$

Les jugements quant à l'usage $\quad 144$

$\begin{array}{ll}\text { CONCLUSION } & 147\end{array}$ 


\section{Le phonème $/ \mathbf{r} /$ en franco-ontarien : réalisations et perceptions}

\section{INTRODUCTION}

Les langues, quelles qu'elles soient, n'échappent pas à la variation et cette variation se manifeste dans toutes les sphères de la langue, dont la sphère phonétique. Celle-là peut être expliquée par l'influence des substrats ou des adstrats linguistiques ${ }^{1}$. Qu'il s'agisse d'étude didactique ou d'étude phonétique, le phonème $/ \mathrm{r}$ / constitue l'un des phonèmes qui mérite une attention particulière. Outre le fait qu'il ait la fréquence d'usage la plus élevée parmi toutes les consonnes du français, le phonème /r/ pose des défis aux apprenants du français langue seconde et langue étrangère et fait montre d'une grande variation ${ }^{2}$ génératrice de jugements sociaux.

Pour certains chercheurs, la consonne roulée apico-alvéolaire [r] est erronément attribuée au latin ${ }^{3}$. En réalité, la consonne roulée apico-alvéolaire était très roulée [ $\mathrm{r}$ ] en ancien français et a probablement été héritée de l'espagnol. Alors que le /r/ du français et le / / / du latin étaient des phonèmes différents qui se trouvaient sous un même archiphonème $/ \mathrm{R} /$, il existe quand même une variante

1. Claude Poirier, « La Langue parlée en Nouvelle-France : vers une convergence des explications », dans Raymond Mougeon, Édouard Beniak (dir.), Les Origines du français québécois, Québec, Presses de l'Université Laval, 1994, p. 237-273; $i d$., "Les Causes de la variation géolinguistique du français en Amérique du Nord : l'éclairage de l'approche comparative », dans Claude Poirier, Aurélien Boivin, Cécyle Trépanier et Claude Verreault (dir.), Langue, espace, société : les variétés du français en Amérique du Nord, Sainte-Foy, Québec, Presses de l'Université Laval, 1994, p. 69-95.

2. Hélène Blondeau, Gilian Sankoff et Anne Charity, « Parcours individuels dans deux changements linguistiques en cours en français montréalais », Revue québécoise de linguistique, vol. 31, $\mathrm{n}^{\circ} 1$, Montréal, Université du Québec à Montréal, 2002, p. 13-38.

3. Le /r/ latin était très roulé alors que le /r/ français avait un roulement beaucoup moins prononcé. 
apico-alvéolaire très roulée $\mathrm{du} / \mathrm{r} /$ en français canadien, que nous retrouvons toujours au nord de l'Ontario. Quoi qu'il en soit, il est certain que la consonne roulée apico-alvéolaire [r] était présente dans l'ancien français et qu'elle a persévéré en moyen français jusqu'au XVII ${ }^{\mathrm{e}}$ siècle dans les centres urbains de même que parmi les personnes âgées, et jusqu'au XVIII ${ }^{e}$ siècle en général pour se faire remplacer, selon certains chercheurs, par la consonne vibrante dorso-uvulaire [R], communément qualifiée de grasseyée ${ }^{4}$ et visant à produire un son consonantique redoublé et, selon d'autres, par la consonne constrictive dorso-vélaire $[\mathrm{s}]^{5}$. Cette consonne redoublée dériverait de la consonne apico-alvéolaire $[\mathrm{r}]$ par un abaissement graduel de l'apex et un mouvement compensatoire du dos de la langue aboutissant au [R] grasseyé parisien ${ }^{6}$.

Le $[\mathrm{R}]$ grasseyé était marqué comme variante de prestige en France au XVIII ${ }^{\mathrm{e}}$ siècle. Et la forte vibration de ce [R] grasseyé a dû s'amenuiser, économie linguistique oblige, pour se transformer, par relâchement de l'uvule, en un [в] fricatif dorso-vélaire.

$\mathrm{Au}$ Québec et ailleurs au Canada français, au début du $\mathrm{XX}^{\mathrm{e}}$ siècle, c'est le [r] roulé, associé à l'élite intellectuelle qui était valorisé. Or, vers la deuxième moitié de ce siècle, cette variante se dévalorise ; elle est abandonnée par l'élite au profit de la variante dorso-vélaire ${ }^{7}$ et elle marque dès lors de plus en plus le langage de la classe populaire et celle des aînés ${ }^{8}$. Ainsi, des perceptions autour de l'usage des deux variantes du $/ \mathrm{r} / \mathrm{se}$

4. Annick Englebert, Introduction à la phonétique historique du français, Bruxelles, de Boeck Supérieur / Duculot, 2009.

5. Georges Straka, Les Sons et les mots. Choix d'études de phonétique et de linguistique, Paris, Klincksieck, 1979, p. 466.

6. Abbé Jean-Pierre Rousselot, « Dictionnaire de la prononciation française (suite) », Revue de phonétique, tome troisième, Paris, 1911, p. 174-175.

7. Luc Ostiguy et Claude Tousignant, Le Français québécois : normes et usages, Montréal, Guérin Universitaire, 1993, p. 164 ; Gabriel Martin, Dictionnaire des onomastismes québécois : les mots issus de nos noms propres, Sherbrooke, Éditions du Fleurdelysé, « Renardeau arctique »1, p. 15.

8. Marty Laforest, "Attitudes, préjugés et opinions sur la langue », dans Claude Verreault, Louis Mercier et Thomas Lavoie (dir), Le Français, une langue à apprivoiser. Textes des conférences prononcées au Musée de la civilisation (Québec, 2000-2001), dans le cadre de l'exposition « Une grande langue : le français dans tous ses états 》, Québec, Presses Université Laval, 2002, p. 84. 
sont traduites par des jugements de valeur ou des clichés qui les marquaient socialement ${ }^{9}$.

$\mathrm{Au}$ début du XXI ${ }^{\mathrm{e}}$ siècle, des réminiscences de l'usage du [r] roulé apico-alvéolaire à l'ouest du Québec se sont vues progressivement commutées par l'usage du [в] dorso-vélaire ${ }^{10}$.

Qu'en est-il de l'usage et de la perception du phonème $/ \mathrm{r} /$ chez les Franco-Ontariens ? À la différence des Français et des Franco-Québécois, les Franco-Ontariens sont une minorité majoritairement bilingue qui vit en contact constant avec l'anglais. Ont-ils les mêmes réalisations phonétique du phonème $/ \mathrm{r} /$ ? Ont-ils les mêmes perceptions ? Cette étude se donne pour objectif d'apporter des réponses à ces questions.

Il s'agit donc d'une étude qui s'inscrit dans le cadre de la phonétique articulatoire et de la sociophonétique. Elle se fonde sur des données socio-démo-linguistiques recueillies par questionnaire auprès de sujets franco-ontariens (hommes et femmes) répartis selon leurs régions de résidence et de provenance, leurs groupes d'âges et leurs catégories socioéconomiques.

\section{I - CONTEXTE}

En français, il y a globalement deux zones articulatoires du phonème $/ \mathrm{r} /{ }^{11}$ : une zone qualifiée d'antérieure, puisque l'articulation se situe à l'avant de la cavité orale, et une zone qualifiée de

9. Denis Dumas, Nos façons de parler : les prononciations en français québécois, Montréal, Presses de l'Université du Québec, 2011.

10. Louis Mercier, «Le Français, une langue qui varie selon les contextes », dans Claude Verreault, Louis Mercier et Thomas Lavoie (dir), Le Français, une langue à apprivoiser, op. cit., p. 46. Les chercheurs ne s'entendent pas sur un symbole et une définition articulatoire unique, puisque Dubois et collaborateurs classent ce symbole sous l'articulation dorso-uvulaire fricative, une infime nuance mais d'importance extrême si on veut être très précis sur un phénomène qui est à peine perceptible à l'oreille. Jean Dubois, Mathée Giacomo, Christiane Marcellesi, Jean-Baptiste Marcellesi et Jean-Pierre Mével, Dictionnaire de la linguistique et des sciences du langage, Paris, Larousse, 1994.

11. Les études portant sur le phonème/r/ ne s'entendent pas sur plusieurs aspects relatifs à ce phonème. Il y a divergence sur le nombre de variantes, sur les représentations phonétiques et, donc, sur la description des unités sous étude. Voir à cet effet, entre autres, Abbé Jean-Pierre Rousselot, op. cit. ; Marty Laforest, op. cit. ; Meike Wernicke-Heinrichs, "The Evolution of French R: A Phonological Perspective », Master of Arts Thesis, Simon Fraser University, 1996. 
postérieure, puisque l'articulation se situe à l'arrière de la cavité orale. Ces deux zones présentent chacune des variations. Dans la zone antérieure, on trouve deux variantes : le [r] apico-alvéolaire roulé et le [r] apico-alvéolaire battu. Dans la zone postérieure, on retrouve le $[\mathrm{b}]$ dorso-vélaire fricatif et le [R] dorso-uvulaire vibrant, appelé aussi le [R] grasseyé ${ }^{2}$. Les variantes de /r/ en français sont normalement utilisées en variation libre, c'est-àdire que leur usage dépend du locuteur (voir figures 1-3) 13 $^{13}$, mais il arrive aussi qu'un locuteur les alterne en fonction de la région de résidence ou de provenance, de la situation ou de l'environnement linguistique dans lequel ce phonème apparait. L'on pourrait alors avoir affaire à des variantes (appelées aussi « allophones ») régionales, situationnelles ou contextuelles ${ }^{14}$.

\section{Figures 1-3}

Formes $\mathrm{du} / \mathrm{r} /$ en français selon les zones articulatoires antérieures et postérieures ${ }^{15}$

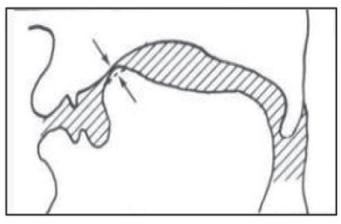

[r] apico-alvéolaire roulé

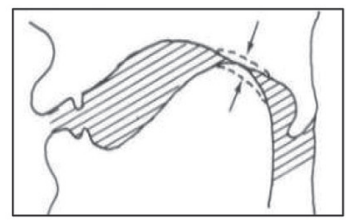

[ь] dorso-vélaire fricatif

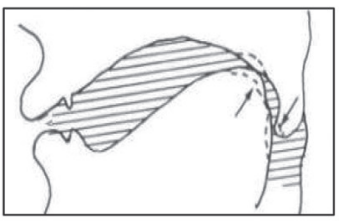

[R] dorso-uvulaire vibrant

Dans les langues du monde, ces variantes correspondent soit à des phonèmes uniques, soit à deux phonèmes différents, soit à un phonème apparenté ou semblable, soit à rien. Ainsi, la variante dorso-vélaire $[\mathrm{K}]$ correspond à un phonème en allemand alors que

12. En contact avec l'anglais, des francophones pourraient produire le son [I] qualifié d'apico-alvéolaire rétroflexe. On le rencontre dans les anglicismes. Dans ce cas, son occurrence en conjonction avec des mots français devient une mesure de l'assimilation. Alex Vanneste, Le Français du XXI siècle : introduction à la francophonie, éléments de phonétique, de phonologie et de morphologie, Bruxelles, Garant, 2005, p. 203.

13. Jean Mazel, Phonétique et phonologie dans l'enseignement du français, Paris, Nathan, 1980, p. 45.

14. Peter Trudgill, « Linguistic change and diffusion : Description and explanation in sociolinguistic dialect », Language in Society, vol. 3, n 2, 1974, p. 215-246.

15. Jean Mazel, loc. cit. 
ce n'est pas le cas de la variante apico-alvéolaire [r]. En arabe, les deux allophones $[\mathrm{r}]$ et $[\mathrm{r}]$ correspondent chacun à un phonème différent : $/ \mathrm{r} /$ et $/ \mathrm{\gamma}^{/ 16}$. En castillan, aux variantes du français [r] et [r] correspondent deux phonèmes distincts : le /r/ roulé et le / / / vibrant et battu. En anglais, l'on entend le son $[\mathrm{r}]$ comme variante régionale concomitante à la variante plus normalisée apicoalvéolaire rétroflexe [I], les deux étant des allophones d'un même phonème /r/. Ces comparaisons laissent entrevoir des problématiques intéressantes dans des domaines comme la didactique du français langue seconde (FLS) ou du français langue étrangère (FLÉ) et de la correction phonétique ${ }^{17}$. En effet, plusieurs études montrent les difficultés qu'ont les étudiants en FLs à apprendre certains phonèmes du français en raison du crible phonologique ${ }^{18}$ de leur langue première (par exemple la confusion des phonèmes $/ 1 /$ et $/ \mathrm{r} /$ par les étudiants japonais et thaïlandais).

En outre, ces sons présentent un intérêt pour la sociolinguistique et la sociophonétique. Par exemple, l'une des questions

16. Même si le son [в] existe en français et en arabe, il relève de deux phonèmes différents. En français, il relève du phonème $/ \mathrm{r} /$ et en arabe du phonème $/ \mathrm{\gamma} /$. Quant au son de l'autre son [r], il relèverait du phonème $/ r /$ en arabe, soit l'équivalent $d u$ phonème français $/ \mathrm{r}$. Autrement dit, il n'y a pas de correspondance complète entre les deux systèmes. Ainsi, au phonème arabe $/ \mathrm{r} /$ correspond le phonème $/ \mathrm{r} / \mathrm{du}$ français, qui a quatre variantes (allophones) : deux antérieures [r] et [r] et deux postérieures [B] et [R]. Quant au phonème arabe / $/ /$, il n'a pas de correspondance phonologique en français, même s'il a une correspondance phonétique, celle du son [ $\mathrm{s}]$. C'est la raison pour laquelle, quand il s'agit d'emprunter des mots arabes, contenant le phonème $/ \mathrm{\gamma} /$, en français, nous avons recours à la translittération. Ce phonème est généralement translittéré par le digraphe «gh ».

17. Monique Callamand, Méthodologie de l'enseignement de la prononciation, Paris, Clé international, 1981 ; Pierre Léon, Phonétisme et prononciations du français, Paris, Nathan Université, 1992 ; Jean-Guy LeBel, Traité de correction phonétique ponctuelle, Québec, Université Laval, CIRAL, 1990; Philippe Munot et FrançoisXavier Nève, Une introduction à la phonétique, Liège, Éd. du CÉFAL, 2002 ; Jacqueline M.-C. Thomas, Luc Bouquiaux et France Cloarec-Heiss, Initiation à la phonétique, Paris, Puf, 1976.

18. Nikolai Sergeyevich Troubetzkoy, Principes de phonologie, Klincksieck, Paris, [édition originale : 1939], $3^{\mathrm{e}}$ éd., 1970. Troubetzkoy définit ainsi le crible phonologique : «Le système phonologique d'une langue est semblable à un crible à travers lequel passe tout ce qui est dit. Seules restent dans le crible les marques phonétiques pertinentes pour individualiser les phonèmes. [...] Chaque homme s'habitue, dès l'enfance, à analyser ainsi ce qui est dit et cette analyse se fait d'une façon tout à fait automatique et inconsciente. Mais, en outre, le système des cribles, qui rend cette analyse possible, est construit différemment dans chaque langue » (p. 54). 
qui suscite de l'intérêt a trait à la manière dont les arabophones emploieraient en arabe des mots d'origine française contenant le phonème $/ \mathrm{r} /$, sachant que la langue arabe contient deux phonèmes distincts $/ \mathrm{r} /$ et $/ \mathrm{\gamma} /$, le premier correspondant aux variantes antérieures et le second aux variantes postérieures du français. Opteraient-ils pour le son [r] ou le son [ $\mathrm{b}]$ ? Et quelle variante utiliseraient-ils quand ils parlent la langue française? Les arabophones ont élu la variante apico-alvéolaire quand il s'agit de parler ou d'écrire en arabe des mots d'origine française, car celle-ci correspond au phonème $/ \mathrm{r}$ / en arabe. Par contre, quand ils parlent le français, ils sont divisés : un groupe opte pour la variante apicoalvéolaire [r], parce qu'il y voit un signe de virilité et qualifie la variante dorso-vélaire d'efféminée ; un autre groupe opte pour la variante dorso-vélaire [ $\mathrm{s}]$, parce qu'il y voit un symbole de culture et de distinction sociale. ${ }^{19}$

Pour les francophones les variantes du phonème $/ \mathrm{r} /$ ont souvent servi de marqueurs sociaux. En effet, nombre d'études ${ }^{20}$ montrent que la réalisation par un locuteur du phonème $/ \mathrm{r} /$ constituerait un marqueur puissant de son appartenance linguistique. En outre, quand le /r/ réalisé par le locuteur diffère du /r/ normatif, une perception d'écart, qui pourrait se doubler d'un sentiment d'étrangeté ou d'une impression d'articulation étrangère, pourrait apparaître chez les locuteurs natifs.

Par ailleurs, dans une étude menée sur l'usage du phonème /r/ rétroflexe ${ }^{21}$ dans les grands magasins de New-York, William

19. Jean-Louis Maume, «L'Apprentissage du français chez les Arabophones maghrébins (diglossie et plurilinguisme en Tunisie) », Langue française, $\mathrm{n}^{\circ} 19$, Paris, Larousse, 1973, p. 90-107 ; Dalila Morsly, «Diversité phonologique du français parlé en Algérie : réalisation de /r/ », Langue française, $n^{\circ}$ 60, Paris, Larousse, 1983, p. 65-72; Aziza Boucherit et Jérôme Lentin, «Les Dialectes féminins dans le monde arabe : des dialectes minoritaires et leur évolution ", LINX (numéro thématique : Genre et langage. Actes du colloque tenu à Paris X-Nanterre les 14-15-16 décembre 1988), nº 21, 1989, p. 17-37.

20. Bernard Tranel, The Sounds of French : An Introduction, Cambridge University Press, 1987 ; Johanne Akpossan, Les Variétés du français : les variantes $d u$ $/ R /$ comme indice de la variation lectale. Variétés, variations et formes du français, Palaiseau, France, 2010. https://lc.cx/wVMY, site consulté le 24 octobre 2017.

21. Ce $[\mathrm{r}]$ apico-alvéolaire est réalisé par le contact de la zone alvéolaire avec l'apex légèrement replié sur lui-même vers le haut. 
$\mathrm{Labov}^{22}$ a montré que la variation de ce phonème en anglais présente une stratification sociale de sorte que plus on monte dans l'échelle sociale, plus le /r/ rétroflexe tend à se maintenir et plus on descend dans l'échelle sociale, plus il tend à s'effacer. Cela va donc dans le même sens que ce que nous avons avancé au début de l'étude et ce qui suivra à l'effet que l'emploi des variantes du phonème /r/ en milieu francophone n'est souvent pas exempt de préjugés.

Le phonème $/ \mathrm{r}$ / a fait l'objet de plusieurs recherches durant le siècle dernier. Les premières études canadiennes se sont intéressées à la répartition des variantes prédominantes du phonème /r/, à savoir la variante antérieure, articulée à l'avant de la cavité orale par l'apex en conjonction avec les alvéoles des dents ([r] apico-alvéolaire), et la variante postérieure articulée à l'arrière de la cavité orale avec le dos de la langue en conjonction avec le vélum ([ь] dorso-vélaire). Selon Poirier $^{23}$ le [r] apico-alvéolaire dominait la région de Montréal alors que le [в] dorso-vélaire dominait la région de Québec. Pour Hull ${ }^{24}$ et $\mathrm{Lucci}^{25}$, les francophones canadiens hors Québec produisent généralement le [r] apico-alvéolaire. À un niveau plus global, Poirier soutient que cette distinction relève du français laurentien, dont font partie le franco-ontarien et le franco-québécois, et du français acadien ${ }^{26}$.

En effet, Selon Poirier $^{27}$, le [r] apico-alvéolaire provient en

22. William Labov, The Social Stratification of English in New York City, Washington, D. C., Center for Applied Linguistics, 1966.

23. Claude Poirier, « Les Causes ... », op. cit., p. 73 ; Yves-Charles Morin, "Les Premiers Immigrants et la prononciation du français au Québec », Revue québécoise de linguistique, vol. $31, \mathrm{n}^{\circ}$ 1, 2002, p. 50.

24. Alexander Hull, « The Franco-Canadian Dialect of Windsor, Ontario : A Preliminary Study », Orbis, 5, 1956, p. 49.

25. Vincent Lucci, Phonologie de l'acadien (parler de la région de Moncton, Nouveau Brunswick, Canada), Montréal, Paris et Bruxelles, Didier, 1972, « Studia Phonetica », 1972, p. 109.

26. Claude Poirier, «Les Causes ... », op. cit. ; voir aussi Hubert Charbonneau et coll., Naissance d'une population. Les Français établis au Canada au XVII siècle, Paris et Montréal, Institut national d'études démographiques et Pum, 1987 ; Geneviève Massignon, Les Parlers français d'Acadie. Enquête linguistique, Paris, Klincksieck, 2 vol., 1962.

27. Claude Poirier, « Les Causes ... », op. cit. 
grande partie des zones rurales de l'ouest de la France et le [в] dorso-vélaire des zones urbaines (en majorité en provenance du centre de la France qui subissait l'influence de la région parisienne). Par ailleurs, la présence de l'aristocratie ainsi que de l'administration française à Québec a favorisé le $[\mathrm{b}]$ dorso-vélaire.

$\mathrm{Au} \mathrm{XVII}{ }^{\mathrm{e}}$ siècle, le $[\mathrm{r}]$ apico-alvéolaire était en nette régression face au [в] dorso-vélaire parmi les membres de l'aristocratie de la région parisienne. Toutefois, il s'est maintenu dans la langue populaire puis dans les parlers régionaux jusqu'au $\mathrm{xx}^{\mathrm{e}}$ siècle. Il s'ensuit que le [r] apico-alvéolaire de Montréal serait ainsi plus attribuable aux français populaires et régionaux de la France ${ }^{28}$.

Au Québec, les années 1950 ont connu une régression rapide du [r] apico-alvéolaire sous l'influence des métropoles de Québec et de Paris et l'apparition de jugements de valeur rattachés à l'usage des deux formes $d$ '/r/ qui ont commencé à attirer l'attention des chercheurs en sociophonétique de même qu'en psychophonétique. À cet effet, Maria Candea et Cyril Trimaille ${ }^{29}$ notent que :

Parmi les précurseurs, Fónagy ${ }^{[30]}[\ldots]$ occupe une place à part au croisement de la phonétique acoustique et de l'analyse des stéréotypes [...], avec l'ambition d'insérer des hypothèses forgées par la psychanalyse. On signalera ainsi la thèse de Boumendil ${ }^{[31]}[\ldots]$ qui, sous sa direction, s'est intéressée aux valeurs symboliques des prononciations parisiennes dont celle $\mathrm{du} / \mathrm{R} /$ dit parisien. Les intuitions de Fonagy, à l'instar de celles de Léon $^{[32]}[\ldots]$, non étayées par des méthodes d'enquête quantitative ou de terrain, sont convoquées pour alimenter

28. Ibid.; Yves-Charles Morin, loc. cit.

29. Maria Candea et Cyril Trimaille, «Phonétique, sociolinguistique, sociophonétique : histoires parallèles et croisements ", Langage et société (Dossier : Sociophonétique du français : genèse, questions et méthodes), vol. 1, nº 151, Paris, Éditions de la Maison des sciences de l'homme, 2015, p. 11. 1983.

30. Ivan Fónagy, La Vive Voix : essais de psycho-phonétique, Paris, Payot,

31. Aliette Boumendil, « Structuration sociale des variations dans la prononciation des Parisiens étudiée du point de vue dynamique », thèse de doctorat, Université de Paris, 1977.

32. Pierre Léon, «Principes et méthodes en phonostylistique», Langue française, $\mathrm{n}^{\circ} 3,1969$, p. $73-84$. 
les hypothèses sur l'indexicalité et le phono-symbolisme de variantes phonétiques en relation avec les communautés de pratiques étudiées bien plus tard par Eckert ${ }^{[33]}[\ldots]$.

Il est vrai qu'à la suite de Labov, la sociolinguistique variationniste s'est essentiellement intéressée, dès le début, aux prononciations et aux liens indexicaux que des variables (indicateurs, marqueurs, stéréotypes) entretiennent avec des dimensions extralinguistiques, sociales, identitaires. Pour comprendre d'où viennent les changements linguistiques et comment ils sont diffusés ou transmis, de nombreuses études des productions phonétiques elles-mêmes, de leur distribution, ont dégagé des patterns de stratification de variables sociolinguistiques (aux plans géographique, social, générationnel, genré), ont établi des corrélations permettant d'attribuer, sur une base statistique, des valeurs sociales à différentes variantes.

Par ailleurs, si la ville de Montréal a subi une certaine influence parisienne, l'Ontario n'a subi que l'influence des français populaires et régionaux. Durant les années 1950, le [r] apico-alvéolaire dominait encore 1'Ontario, contrairement à ce qu'il en était à Montréal. En effet, Yves-Charles Morin ${ }^{34}$ rapporte que Claude Poirier $^{[35]}$ conclut

à partir d'évaluations démographiques indirectes, [...] que « la région de Québec a reçu proportionnellement plus de colons venant du centre de la France (région dominée par l'influence parisienne) et du nord-ouest que celle de Montréal [qui a reçu plus d'immigrants ruraux de l'ouest de la France] » et qu'ainsi «l'influence des ruraux a été sensiblement plus forte dans la région de Montréal ». Ce serait cette différence de peuplement, combinée à « une tendance normalisatrice découlant de la présence de fonctionnaires de l'administration française [...] en poste à Québec », qui expliquerait la différence entre l'articulation apicale [r] du $\mathrm{r}$ à Montréal et son articulation postérieure (le plus souvent l'uvulaire [в]) à Québec. Notons que dans cette argumentation, c'est moins l'origine géogra-

33. Penny Eckert, "Affect, Sound Symbolism, and Variation », University of Pennsylvania Working Papers in Linguistics, vol. 15, $\mathrm{n}^{\circ}$ 2, 2010, p. 70-80.

34. Yves-Charles Morin, op. cit., p. 50.

35. Claude Poirier, « Les Causes ... », op. cit., p. 74-75. 
phique des colons qui est en cause que leur origine sociale : c'est surtout l'opposition entre villes et campagnes qui est déterminante. Car même les colons ruraux venant du centre de la France, toute empreinte que soit cette région de la norme parisienne, devaient alors avoir des [r] apicaux - c'est en tout cas l'articulation qu'utilisaient dans ma jeunesse, les aînés de mon propre terroir briard, ceci à moins de cent kilomètres à l'est de Notre-Dame de Paris, ainsi que les témoins de Fondet $[\ldots]^{36}$, à une distance de moins de vingt-cinq kilomètres au sud de la même cathédrale.

\section{II - OBJECTIFS DE L'ÉTUDE}

Compte tenu de l'histoire du phonème /r/ et des conditions sociolinguistiques qui ont marqué son étude, notre recherche poursuit quatre objectifs :

1. Étudier la répartition des articulations antérieures et postérieures du phonème $/ \mathrm{r} /$ en franco-ontarien ;

2. déterminer les facteurs qui influencent la production de la variante antérieure ou de la variante postérieure ;

3. déterminer la préférence des locuteurs pour une variante plutôt que l'autre ;

4. déterminer les raisons qui sous-tendent les préférences des locuteurs pour une variante ou pour l'autre.

\section{Problématique}

Suite à ces objectifs, il nous est possible maintenant de poser les questions de recherche qui s'imposent et pour lesquelles nous tenterons de trouver des réponses.

1. Qu'en est-il des usages du phonème / $\mathrm{r} /$ en franco-ontarien ?

2. Quelles sont les influences de l'état actuel des usages du phonème $/ \mathrm{r} /$ en Ontario ?

3. Quelles sont les perceptions des usages du phonème $/ \mathrm{r} /$ en Ontario compte tenu des jugements esthétiques qui prévalent à peu près partout?

36. Claire Fondet, Dialectologie de l'Essonne et de ses environs immédiats, Lille, Atelier de reproduction des thèses, Université de Lille III et Paris, Champion (diffusion), 1980. 


\section{Hypothèses}

Pour des motifs sociolinguistiques usuels, nos hypothèses sont les suivantes.

H1. Les femmes adopteront plus le [в] dorso-vélaire que les hommes.

H2. Les plus jeunes adopteront plus le [в] dorso-vélaire que les plus vieux.

H3. Les plus jeunes seront plus portés à alterner le $[\mathrm{b}]$ dorsovélaire et le /r/ apico-alvéolaire.

H4. Plus les sujets sont instruits, plus ils adopteront le [в] dorso-vélaire.

H5. Plus les sujets sont instruits, moins ils alterneront les variantes du phonème $/ \mathrm{r} /$.

H6. Les habitudes articulatoires des parents n'auront pas d'influence sur la forme que prendra le phonème $/ \mathrm{r} /$ chez les enfants.

H7. La région où le locuteur a passé les dix premières années de sa vie déterminera la forme que prendra le phonème $/ \mathrm{r} /$.

H8. Le phonème / $r$ / subira une influence normalisatrice vers le [в] dorso-vélaire.

H9. L'influence normalisatrice sera plus marquée dans le centre et dans l'est de l'Ontario.

H10. Les jugements naturels seront plus prononcés que les jugements esthétiques quant aux usages du phonème $/ \mathrm{r} /$.

\section{Méthodologie}

L'étude s'est déroulée en Ontario (voir figure $4^{37}$ ). Elle a couvert le Nord-Est, l'Est et le Centre de 1'Ontario. Le Nord-Ouest et le Sud-Ouest, où la proportion des francophones est moins importante, ne nous ont permis de recruter qu'un seul sujet par région pour l'enquête. Nous avons donc élu de ne pas les inclure dans l'étude. Par ailleurs, un autre sujet a dû être éliminé, parce qu'il ne correspondait pas au critère de résidence en Ontario durant les dix premières années de sa vie.

37. Assemblée de la francophonie de l'Ontario, 2018, https://lc.cx/dqgR, site consulté le 9 mars 2018. 


\section{Figure 4}

Régions de l'Ontario

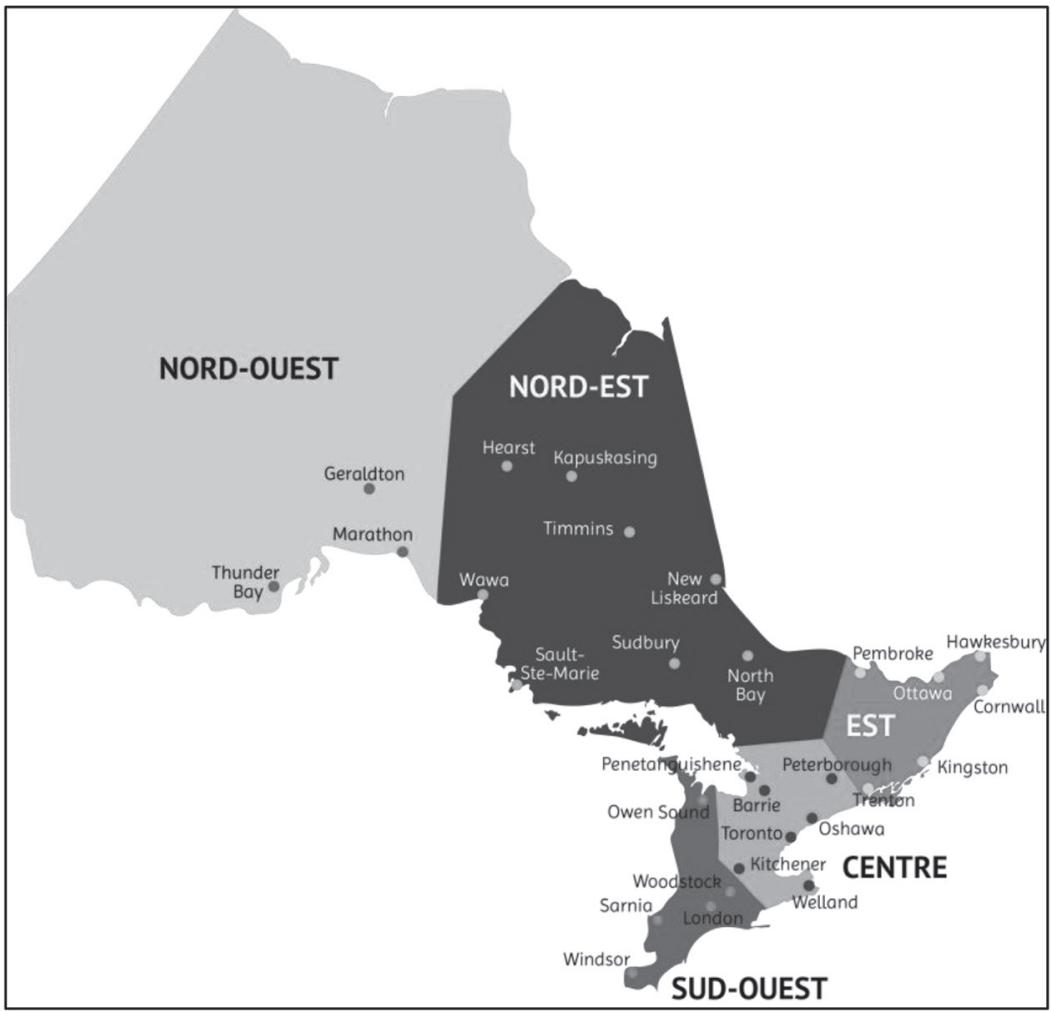

Nous avons retenu 120 sujets $^{38}$ (32 hommes et 88 femmes), répartis selon l'âge comme suit : 71 sujets âgés de 15 à 19 ans, 34 de 20 à 30 ans, 8 de 31 à 50 ans, 7 de plus de 51 ans. Sur ces 120 sujets, 103 ont dit avoir une résidence stable quant aux dix premières années de leur vie (soit 82 du Nord-Est, 11 de l'Est et $10 \mathrm{du}$ Centre). En ce qui concerne la résidence officielle des sujets durant les dix dernières années, 118 sur 120 ont déclaré avoir la même résidence (soit 105 résidents du Nord-Est, 5 de l'Est et 8 du Centre).

38. Nous tenons à remercier notre étudiante Valérie Gauthier qui a bien voulu recruter ses sujets de mémoire de spécialisation pour les fins de notre étude. Nous tenons aussi à remercier nos collègues Amélie Hien et Valérie Raymond qui ont eu la gentillesse de faire circuler le questionnaire parmi leurs étudiantes et étudiants qui ont bien accepté de participer à l'étude. Que tous les participants trouvent ici notre appréciation et reconnaissance. 
Le questionnaire s'est enquis du niveau scolaire le plus élevé atteint par les sujets pour voir si cette variable affectait la forme $\mathrm{du} / \mathrm{r} /$ prononcé. À cet effet, sur le plan du niveau d'instruction, les sujets étaient répartis comme suit : 2 de niveau primaire, 75 de niveau secondaire (première année d'université), 15 de niveau collégial, 21 de niveau de baccalauréat, 3 de niveau de maîtrise, 3 de niveau de doctorat et un répondant n'a pas donné de réponse à cette question.

Les sujets devaient répondre à des questions concernant les variantes de /r/ qu'ils utilisent dans leur parler, à savoir s'ils utilisent la variante qui se réalise avec la pointe de la langue (soit la variante antérieure apico-alvéolaire, que celle-ci soit roulée, battue ou rétroflexe) ou celle qui se réalise dans la gorge (soit la variante postérieure, que celle-ci soit dorso-vélaire ou dorsouvulaire), ou s'ils alternent les deux variantes. Pour les besoins de notre recherche, nous n'envisageons étudier que l'antériorité ou la postériorité du phonème $/ \mathrm{r} /$.

En outre, les sujets devaient aussi indiquer s'ils avaient une préférence pour une variante ou l'autre en choisissant parmi un certain nombre de jugements pour déterminer leurs perceptions de la variante qu'ils utilisent de celle qu'ils n'utilisent pas. Une liste de jugements a été obtenue au préalable grâce à une pré-enquête, auprès de 30 sujets, dans laquelle nous leur avons demandé d'écrire littéralement toutes les raisons qu'ils ont de préférer une variante et toutes les raisons qu'ils ont de ne pas préférer l'autre variante. Suite à cette préenquête, nous avons dressé, à partir des jugements littéraux recueillis, une liste de jugements que nous avons classés en deux catégories, soit une catégorie naturelle qui implique des jugements de facilité, d'aisance articulatoire et de modèle présent dans la famille ou dans le milieu, et une catégorie esthétique qui invoque la beauté ou la laideur, la distinction ou la non-distinction sociale. Cette liste a été incluse dans le questionnaire de l'enquête à proprement parler et les sujets devaient cocher un ou plusieurs jugements. Cela dit, nous avons ajouté un jugement « autre » pour anticiper les cas où il y aurait d'autres 
jugements qui n'avaient pas été soulevés dans la préenquête, ce qui ne fut pas le cas.

Par ailleurs, afin de déterminer si le modèle parental et le modèle sociétal dans les dix premières années de la vie et dans les dix dernières années avaient un effet ou non sur la variante utilisée, nous avons demandé aux sujets d'indiquer les variantes pratiquées par leurs mères et leurs pères, de même que la région dans laquelle ils ont vécu le plus longtemps durant les dix premières années de leur vie et la région de résidence officielle.

\section{III - ANALYSE ET INTERPRÉTATION DES RÉSULTATS}

Partant du constat général que, en ce moment précis de l'évolution $\mathrm{du}$ franco-ontarien en matière du phonème $/ \mathrm{r} /$, l'Ontario privilégie le [в] dorsal (51,7\%), que l'alternance entre le [r] apical et le [в] dorsal se place loin au deuxième rang $(29,2 \%)$ et que le [r] apical se classe bon dernier (19,2\%), la figure 5, montre clairement cette tendance vers la normalisation ainsi qu'un niveau important d'alternance entre les deux variantes de /r/, ce qui impose une réflexion sur son statut, son état et son avenir.

\section{Figure 5}

Portrait actuel de l'usage du /r/ en Ontario (en pourcentage)

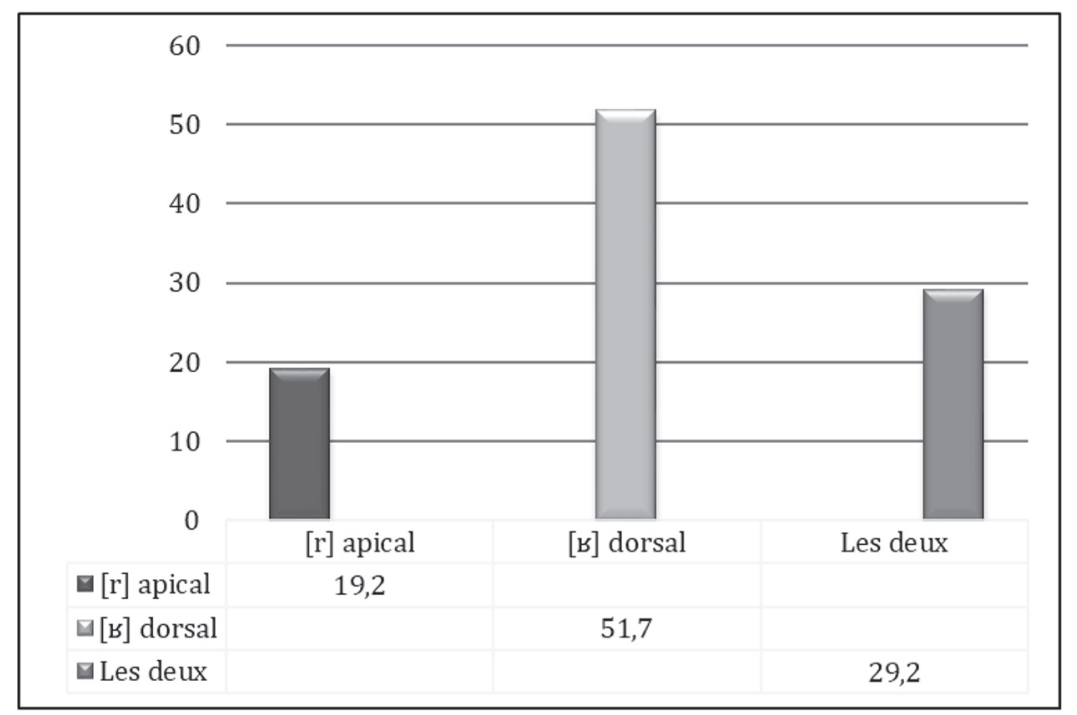




\section{Effet du sexe}

Toutes les études montrent que les femmes sont plus portées que les hommes à adopter de nouvelles habitudes et attitudes, de même que des formes plus standardisées ${ }^{39}$. C'est dans cet esprit que nous avons posé la première hypothèse à savoir que les femmes adopteront plus que les hommes le [в] dorsal.

Les résultats ne confirment pas cette hypothèse ${ }^{40}$. En effet, la figure 6 montre que 48,28\% des femmes utilisent le [в] dorsal contre $62,50 \%$ des hommes. Nous remarquons aussi que ce sont les femmes qui alternent le plus les deux $/ \mathrm{r} /$, soit $31,03 \%$ contre $21,88 \%$ des hommes. Enfin, compte tenu des études antérieures sur l'usage du /r/ jusqu'aux années 1970 qui indiquaient encore une prévalence $\mathrm{du}[\mathrm{r}]$ apico-alvéolaire ${ }^{41}$, nous remarquons que ce [r] a perdu du terrain, puisque son niveau de maintien a atteint $15,62 \%$ chez les hommes et 20,69 \% chez les femmes. Donc, il est clair qu'il y a un mouvement général vers le $[\mathrm{b}]$ dorsal et un mouvement à cheval entre le $[\mathrm{r}]$ apical et le $[\mathrm{b}]$ dorsal. Mais nous ne pouvons pas à ce point prévoir si cette alternance va se résorber au profit du [в] dorsal ou si une distribution complémentaire se créera entre les deux variantes de $/ \mathrm{r} /$.

Nous avons ensuite fait une analyse pour voir si l'usage d'une variante plutôt qu'une autre selon le sexe correspond aussi à une préférence fondée sur le sexe. Cette analyse (figure 7) a permis de voir que les hommes préfèrent plus le [в] dorsal $(41,94 \%)$ que ne le font les femmes $(31,40 \%)$, mais que cette différence

39. Penelope Eckert, « The whole woman: Sex and gender differences in variation ", Language Variation and Change, vol. 1, p. 245-268, 1989, Cambridge University Press ; William Labov, Sociolinguistic patterns, Philadelphia, University of Pennsylvania Press, 1972 ; id., "The intersection of sex and social class in the course of linguistic change », Language Variation and Change, vol. 2, n 2, p. 205-254, 1990 , Cambridge University Press. Dans cet article, William Labov indique que les femmes sont plus portées que les hommes à adopter des attitudes innovatrices: "Two general principles of sexual differentiation emerge from previous sociolinguistic studies : that men use a higher frequency of nonstandard forms than women in stable situations, and that women are generally the innovators in linguistic change » (p. 205).

40. $\chi_{(2)}^{2}=0,80 ; \mathrm{p}=0,669$.

41. Claude Poirier, «Les Causes... », op. cit.; Charles Morin, op. cit. ; Vincent Lucci, op. cit. 
Figure 6

Le /r/ prononcé selon le sexe (en pourcentage)

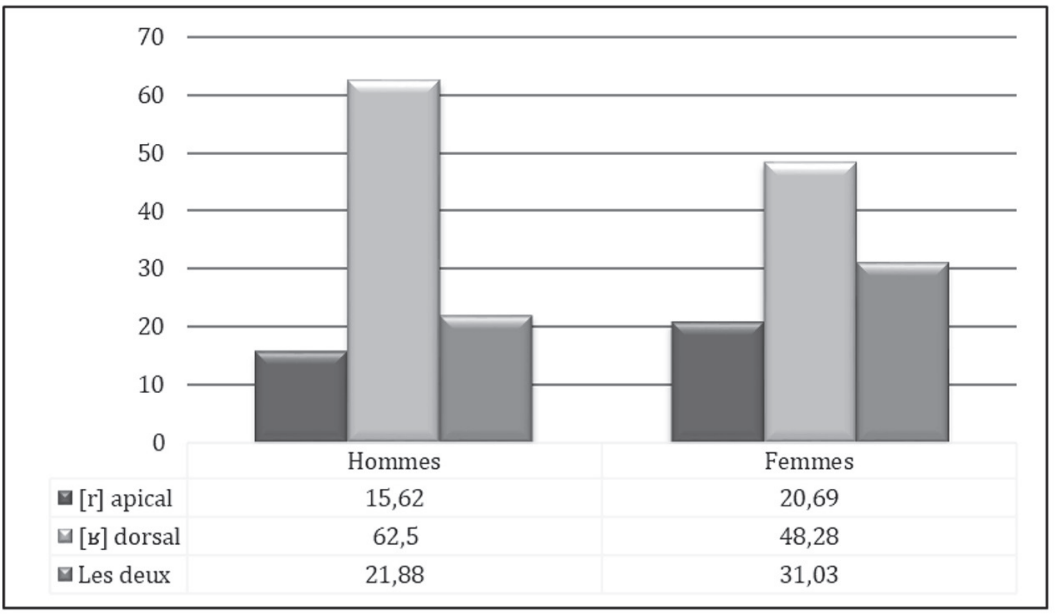

n'est pas significative. Nous remarquons par contre un haut niveau de non-préférence et pour la variante apicale et pour la variante dorsale, soit 51,61\% chez les hommes et 54,65 \% chez les femmes. Ainsi, les femmes se rattrapent quelque peu du fait qu'elles adoptent moins le [ $\mathrm{b}$ ] dorsal que les hommes, en ayant une plus grande acceptation des deux variantes du phonème $/ \mathrm{r} /$. Comme les recherches montrent que les femmes sont d'habitude plus portées que les hommes vers la modernisation, le changement et la standardisation, phénomène traité sous l'hypercorrection chez William Labov, ce résultat vient mettre un bémol sur cette caractéristique féminine et ouvre la porte à une plus grande tolérance chez les femmes envers les deux variantes du phonème $/ \mathrm{r}$ / qui deviennent donc plus acceptables alors que, dans un passé non lointain, elles étaient marquées socialement, et elles le sont encore au Québec et dans plusieurs régions du monde.

\section{Effet de l'âge}

Les changements linguistiques naissent naturellement parmi les jeunes générations et ce sont les générations les plus âgées qui résistent le plus au changement, d'où la variation intergénération- 
Figure 7

Le /r/ préféré selon le sexe (en pourcentage)

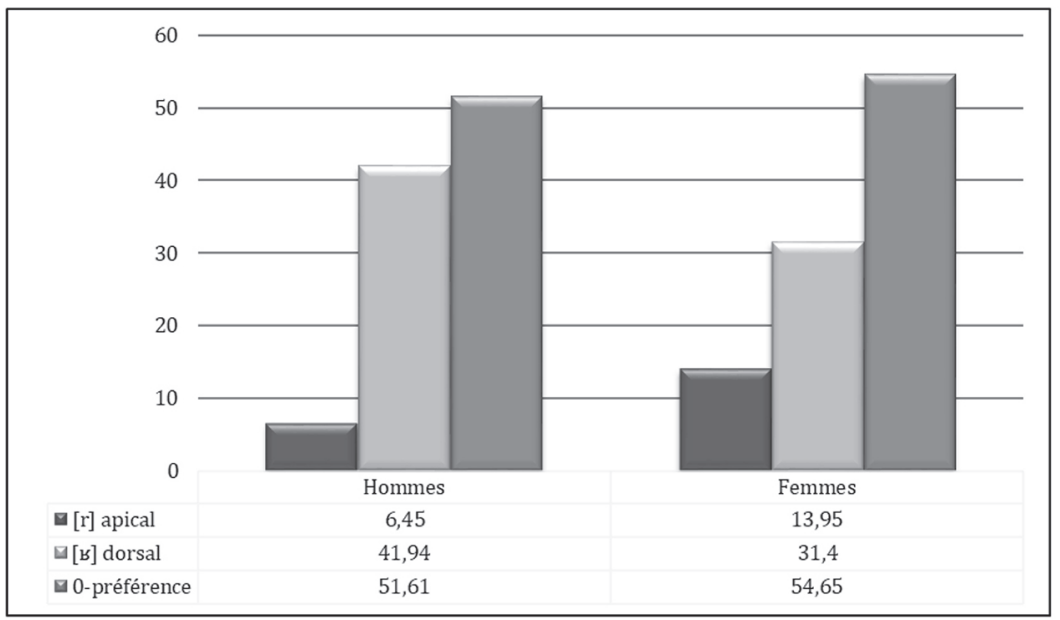

nelle ou diachronique ${ }^{42}$. Les projets d'aménagement du corpus qui portent soit sur le lexique général, soit sur la grammaire, soit sur l'orthographe visent à régulariser ce qui a pris racine dans l'usage auprès des locuteurs de la langue. Plusieurs de ces cas d'aménagement répondent à des innovations nées parmi les jeunes. C'est dans cette perspective que nous avons posé la deuxième hypothèse à l'effet que les plus jeunes adopteront davantage le [ $\mathrm{B}]$ dorsovélaire que les plus vieux, et la troisième hypothèse qui anticipe une plus grande alternance des deux variantes de /r/ chez les plus jeunes que chez les plus vieux.

Notre analyse (figure 8) montre, en effet, que les jeunes tendent à adopter le [в] dorsal (47,89\%), mais, en cela, ils ne sont pas les précurseurs du changement normalisant. Une comparaison intergénérationnelle en synchronie montre que ce sont plutôt les plus âgés qui se sont presque complètement départis de leur [r] apical au profit du [в] dorsal $(85,71 \%)$. Chez les sujets âgés de 15 à 30 ans, nous remarquons un changement marqué vers le [ $\mathrm{B}$ ]

42. Gudrun Ledegen, Isabelle Léglise, "Variations et changements linguistiques », dans S. Wharton et J. Simonin, Sociolinguistique des langues en contact, Ens Éditions, 2013, p. 315-329. 
Figure 8

Le /r/ prononcé selon l'âge (en pourcentage)

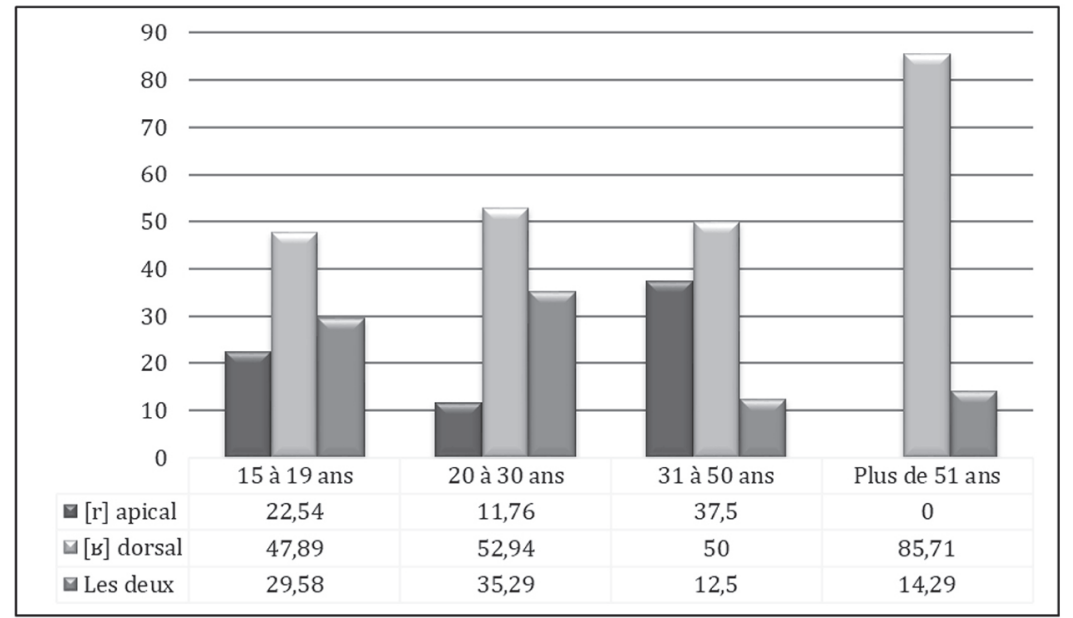

dorsal $(52,94 \%)$, mais nous remarquons aussi que bon nombre d'entre eux alternent entre les deux variantes plus que ne le font les sujets âgés de plus de 31 ans $(29,58 \%$ chez les 15 à 19 ans et $35,29 \%$ chez les 20 à 30 ans contre $12,5 \%$ chez les 31 à 50 ans et $14,29 \%$ chez les plus de 51 ans). Mais, malgré la présence d'une tendance marquée vers l'usage $\mathrm{du}[\boldsymbol{\mathrm { r }}]$ dorsal et l'alternance des deux variantes du phonème $/ \mathrm{r} /$, les différences dues à l'âge ne s'avèrent pas significatives dans notre corpus ${ }^{43}$.

Nous avons exploré le rapport de l'âge à la préférence accordée aux variantes de /r/ (figure 9) pour voir si cela diffère du rapport à l'usage de ces variantes et nous avons pu constater que, dans l'ensemble, 51,67\% des sujets utilisent le [в] dorsal alors que seulement $34,75 \%$ lui accordent une préférence (soit, des plus jeunes aux plus âgés, 33,8 \%, 36,36\%, 28,57 \% et 42,86\%). Nous avons en outre constaté que, dans l'ensemble, 29, $17 \%$ des sujets alternent entre les deux variantes de /r/ alors que 53,39\% d'entre eux n'avaient aucune préférence et, par conséquent, n'auraient pas de jugement à formuler à l'endroit de l'une ou l'autre des deux variantes.

43. $\chi_{(2)}^{2}=0,83 ; p=0,660$. 
Figure 9

Le /r/ préféré selon l'âge (en pourcentage)

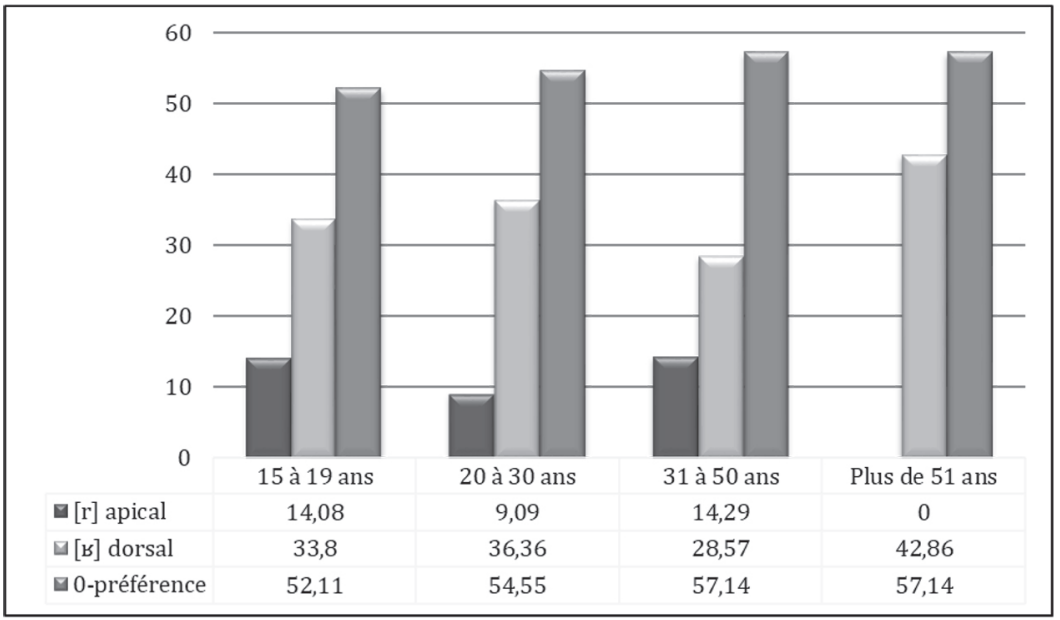

\section{Effet du niveau d'instruction}

Afin de voir si le niveau d'instruction joue un rôle ou non dans l'usage et dans la préférence d'une variante du phonème /r/, nous avons posé les quatrième et cinquième hypothèses à l'effet que, plus on est instruit, plus on adopte le [в] dorsal et moins on alterne les variantes de $/ \mathrm{r} /$. Les résultats en ce qui concerne l'effet du niveau d'instruction sur l'usage du phonème /r/ ne laissent pas entrevoir des tendances différentes de celles que nous avons observées ci-dessus. La figure 10 montre une forte propension à l'usage du [в] dorsal peu importe le niveau d'instruction. Cette tendance semble s'accentuer avec la progression dans le niveau d'instruction. Dans l'ensemble, 51,67 \% des sujets produisent le [в] dorsal, alors que 29,17\% d'entre eux alternent entre les deux variantes du phonème $/ \mathrm{r} /$ et seulement $19,17 \%$ continuent à utiliser le [r] apical. Toutefois, la différence entre les divers groupes ne semble ainsi pas significative.

En ce qui a trait à la préférence d'une variante du phonème /r/ selon le niveau d'instruction, la figure 11 montre la même tendance relevée sous l'effet de l'âge. Autrement dit, les résultats sur l'effet de l'âge et sur celui du niveau d'instruction sont cor- 
Figure 10

Le /r/ prononcé selon le niveau d'instruction (en pourcentage)

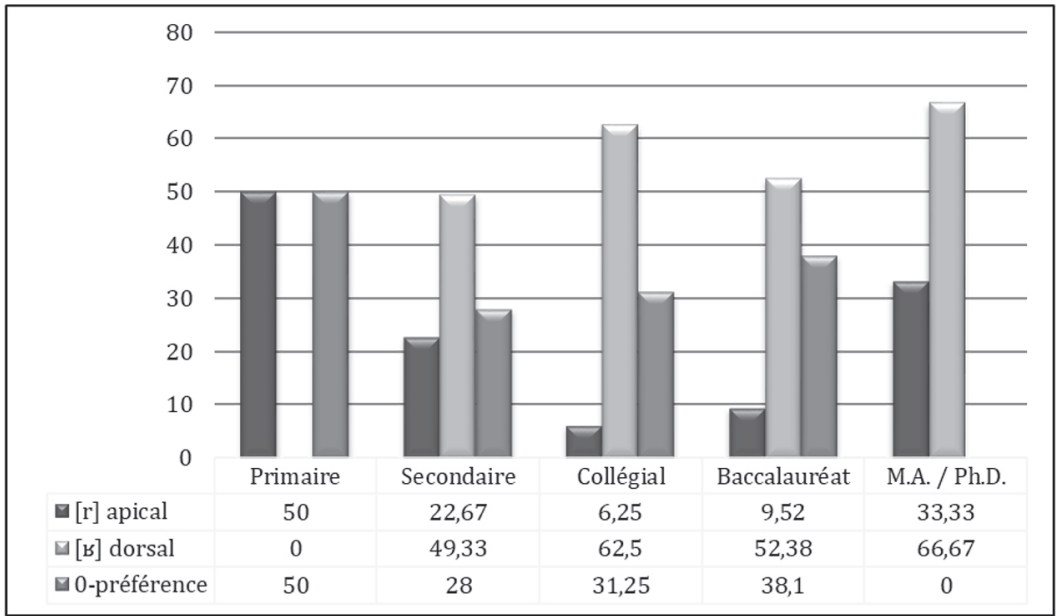

rélés en ce qu'ils ne permettent pas de voir un effet significatif ni sur l'usage ni sur la préférence, mais qu'ils sont aussi corrélés quant à la tendance à l'usage du [ $]$ dorsal, à l'alternance entre les deux variantes du phonème $/ \mathrm{r} /$ et à une plus forte tendance vers l'absence de préférences envers l'une ou l'autre des deux variantes.

\section{Figure 11}

Le /r/ préféré selon le niveau d'instruction (en pourcentage)

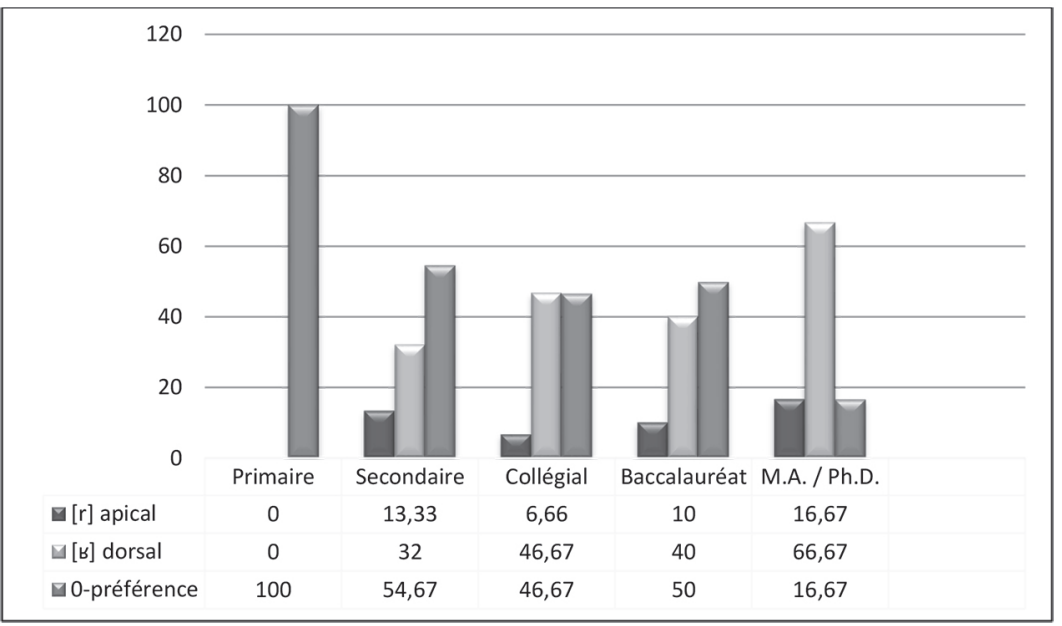




\section{Effet des habitudes articulatoires des parents}

Même si les enfants passent beaucoup de temps avec leurs parents durant les premières années de leur vie et que nous nous attendrions, de ce fait, à ce qu'ils développent les habitudes articulatoires de leurs parents, nous constatons souvent de grandes différences entre les habitudes articulatoires développées par les enfants d'une même famille. En outre, nous constatons souvent des habitudes articulatoires qui ne reflètent pas toujours celles des parents. Ce constat devient d'autant plus percutant quand la réalisation du phonème $/ \mathrm{r} /$ des enfants est totalement à l'opposé de celle des parents ${ }^{44}$. Cela ne peut s'expliquer que par l'influence de facteurs environnementaux, tels les pairs, les éducateurs et éducatrices, la gardienne... Ainsi, notre sixième hypothèse stipule que les habitudes articulatoires des parents n'auront pas d'influence sur la forme que prendra le phonème $/ \mathrm{r} / \mathrm{chez}$ les enfants. Pour ce faire, nous avons examiné l'effet des modèles maternel et paternel de prononciation du /r/ sur l'usage et sur la préférence d'une variante $\mathrm{du}$ phonème $/ \mathrm{r} / \mathrm{chez}$ les enfants. Contrairement à nos attentes, les résultats (figures 12,13,14) ont permis de constater un effet significatif des habitudes articulatoires des parents sur l'usage $\mathrm{du} / \mathrm{r} / \mathrm{chez}$ les enfants. Nous remarquons notamment un résultat de 44,2\% pour l'usage du [в] dorsal et de $15 \%$ pour l'usage des deux variantes de $/ \mathrm{r} /$ en ce qui concerne l'influence du $/ \mathrm{r} /$ du père ${ }^{45}$. De plus, nous remarquons un résultat de $34,2 \%$ pour

44. La théorie des systèmes écologiques soutient que l'environnement de l'enfant affecte la façon dont il grandit et se développe sur tous les plans, incluant le niveau langagier. Différents niveaux ou systèmes environnementaux influencent le développement de l'enfant. Les relations immédiates avec sa famille ou les aidants naturels, la garderie, l'école servent de modèles. Mais la façon dont l'enfant agit ou réagit avec ces personnes aura une incidence sur la façon dont il sera traité en retour. Le tempérament de l'enfant influence les relations et crée des différences entre les enfants d'une même famille. Cela pourrait expliquer en partie que des enfants de la même famille aient des habitudes articulatoires différentes, pouvant différer de celles des parents. Voir Noam Chomsky, "A Review of Verbal Behavior by B.F. Skinner », Language, $\mathrm{n}^{\circ} 35,1959$, p. 26-58 ; Steven Pinker, Language learnability and language development, Cambridge, Massachusetts, Harvard University Press, 1984 ; Urie Bronfenbrenner, The ecologyof human development: Experiments bynature and design, Cambridge, Massachusetts, and London, Harvard University Press, 1979 ; Lev S. Vygotski, Pensée et langage, Paris, Éditions Sociales, 1985.

45. $\chi_{(4)}^{2}=44,95 ; \mathrm{p}=0,001$. 
l'usage du [в] dorsal et de 17,5\% pour l'usage des deux variantes $\mathrm{de} / \mathrm{r} / \mathrm{en}$ ce qui concerne l'influence $\mathrm{du} / \mathrm{r} / \mathrm{de}$ la mère ${ }^{46}$. Donc, il y a un effet significatif pour chacun des modèles parentaux de même qu'un effet significatif combiné.

\section{Figure 12}

Usage $\mathrm{du} / \mathrm{r} /$ selon le $/ \mathrm{r} /$ du père (en pourcentage)

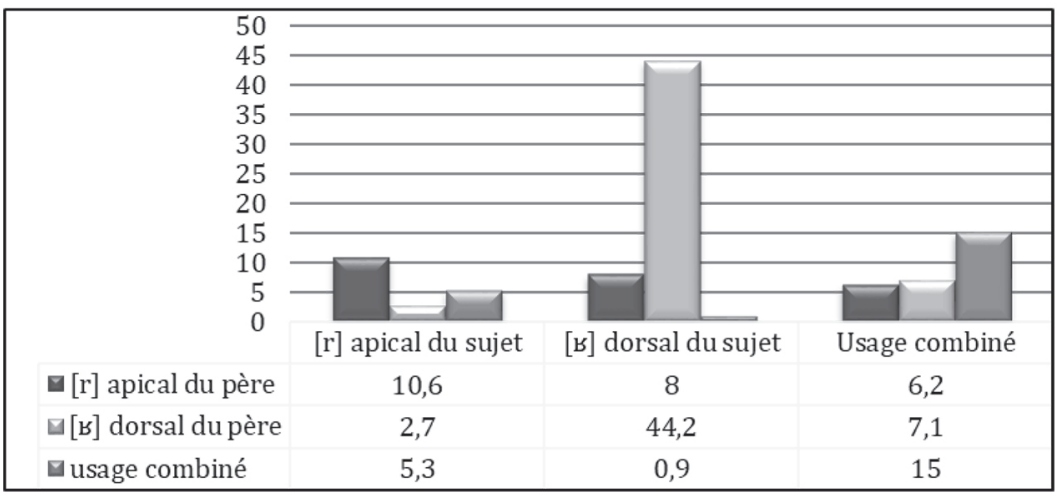

Figure 13

Usage $\mathrm{du} / \mathrm{r} /$ selon le $/ \mathrm{r} / \mathrm{de}$ la mère (en pourcentage)

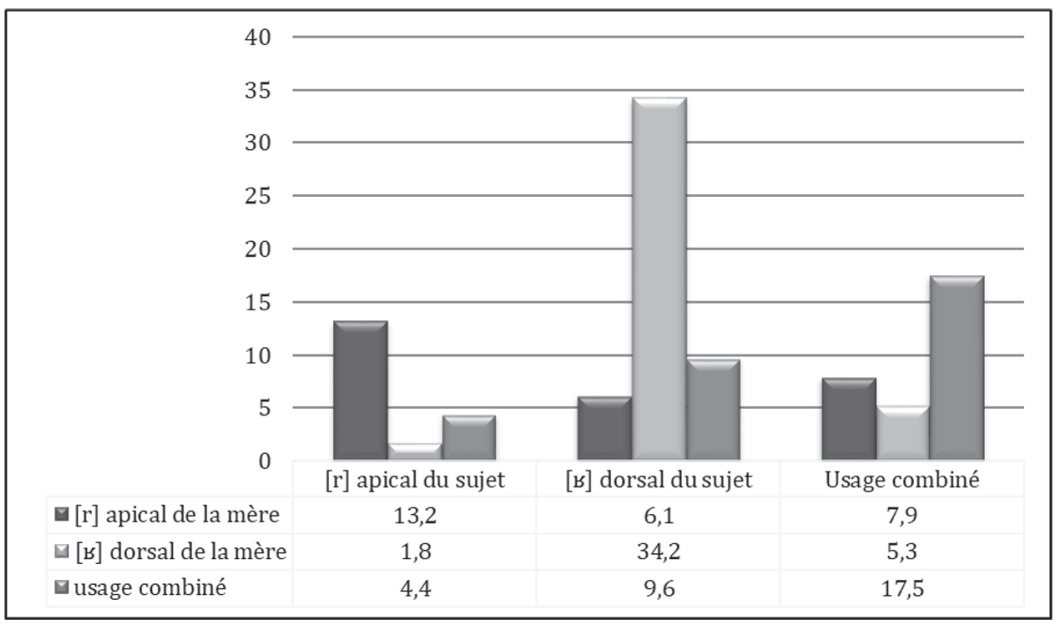

46. $\chi_{(4)}^{2}=39,20 ; \mathrm{p}=0,001$. 
Figure 14

Usage du /r/ selon le /r/ des parents (en pourcentage)

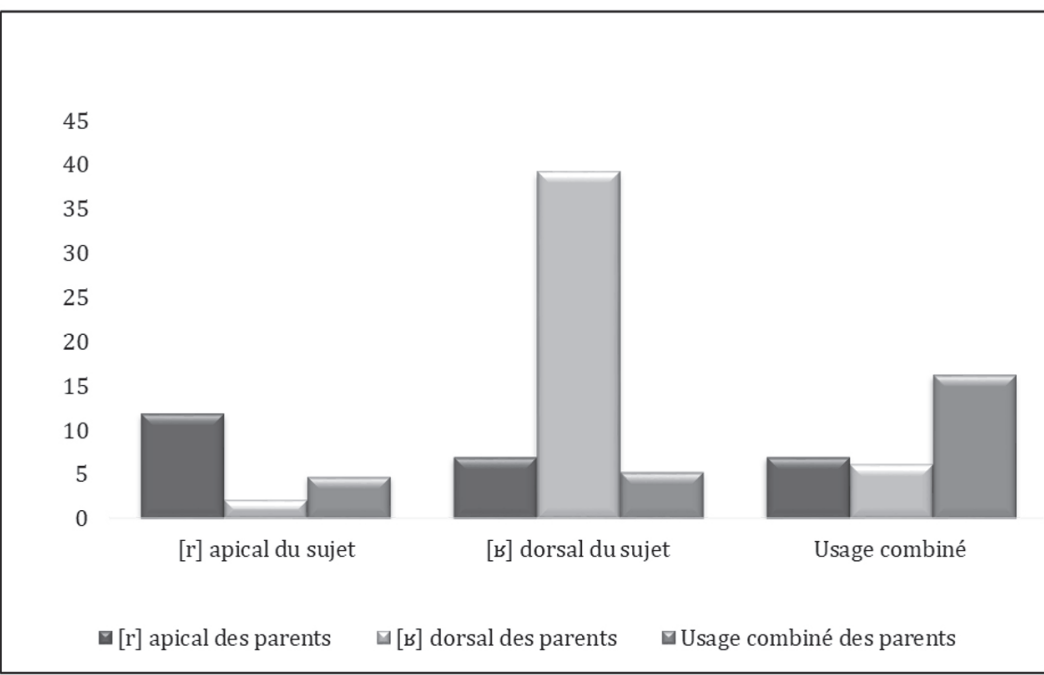

\section{Effet de la région où l'on a vécu le plus longtemps}

Malgré les débats et les polémiques qui animent les spécialistes sur la notion de période critique pour le développement de la langue maternelle (on parle de période sensible pour l'acquisition de la langue seconde), il est admis dans les milieux de recherche en psychologie du développement et en biologie développementale ${ }^{47}$, et nous en convenons, que les dix premières années de vie de l'enfant constituent des années critiques pour l'acquisition des servitudes de la langue, mais surtout pour l'achèvement de certains processus langagiers qui ont besoin de temps pour se stabiliser, en l'occurrence l'usage du phonème $/ \mathrm{r} /$.

C'est dans cette perspective que la septième hypothèse à l'effet que le lieu où l'enfant a vécu le plus longtemps durant les dix premières années de sa vie serait un facteur déterminant de la forme que prendra le phonème $/ \mathrm{r} / \mathrm{chez}$ celui-ci.

47. Eric Heinz Lenneberg, Biological Foundations of Language, New York, Wiley, 1967 ; d'après Lenneberg, c'est vers l'âge de deux ans que la période critique pour l'acquisition du langage commence. En outre, il soutient que cette période se poursuit jusqu'à la période de puberté, durant laquelle le cerveau dispose encore d'une grande plasticité neuronale de nature à accommoder les apprentissages. 
Bien que la tendance générale plaide en faveur d'un effet du lieu où l'on a vécu le plus longtemps durant les dix premières années de sa vie, l'analyse des résultats n'a pas permis de conclure que cette tendance soit de nature significative ${ }^{48}$.

Par ailleurs, notre huitième hypothèse prévoit que le phonème /r/ subira une influence normalisatrice vers le [в] dorso-vélaire. Comme on peut le constater dans la figure 15, le [в] dorso-vélaire semble très en avance et annonce ainsi un mouvement ferme vers la normalisation. Toutefois, il y a aussi l'alternance des deux variantes de /r/ qui nous interpelle vers de nouvelles perspectives de recherche.

Par ailleurs, la neuvième hypothèse prévoit que l'influence normalisatrice sera plus marquée dans le Centre et dans l'Est de l'Ontario. En effet, compte tenu du nombre plus important de francophones dans ces régions, de la présence d'institutions gouvernementales, provinciales et fédérales et d'instances éducationnelles et culturelles, ainsi que des contacts plus avancés avec la francophonie internationale, il appert que ces facteurs forment des forces normalisatrices incontournables.

Force est de constater que les résultats semblent confirmer les huitième et neuvième hypothèses puisque le $[\mathrm{r}]$ dorso-vélaire l'emporte dans toutes les régions de l'Ontario (exceptées celles exclues pour manque de données) et que ce gain semble plus accentué dans le Centre et dans l'Est. Cette disproportion régionale quant à l'usage du $[\mathrm{b}]$ dorso-vélaire pourrait être attribuable à la présence des grands centres urbains abritant Ottawa, la capitale nationale du Canada, et Toronto, la capitale politique et économique de l'Ontario et métropole du Canada. Les figures 15 et 16 montrent l'état de l'usage du/r/ selon la région où le sujet a vécu le plus longtemps ses dix premières années de vie et selon la région de résidence officielle.

La comparaison des deux figures, qui se situe sur le plan temporel, montre une nette tendance à la normalisation puisque le niveau d'usage du [в] dorso-vélaire semble en croissance au

48. $\chi_{(10)}^{2}=10,61 ; p=0,389$. 
Figure 15

Usage $\mathrm{du} / \mathrm{r} /$ selon la région où on a vécu le plus longtemps durant les dix premières années de sa vie (en pourcentage)

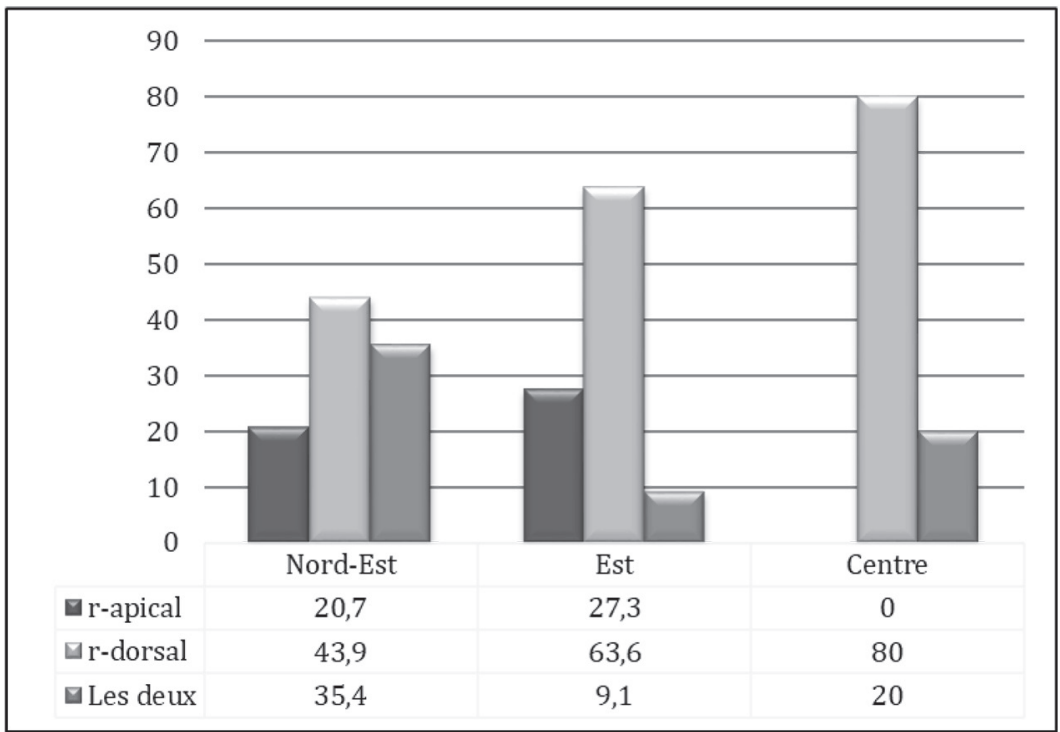

Figure 16

Usage $\mathrm{du} / \mathrm{r} /$ selon la région de résidence officielle (en pourcentage)

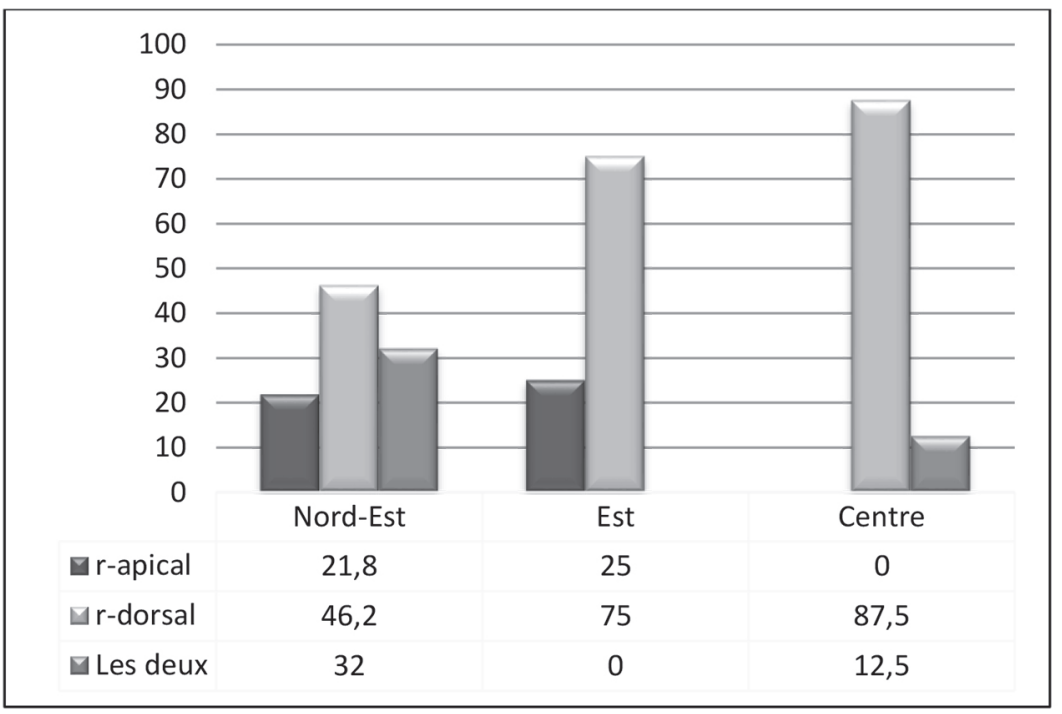


point que le $[\mathrm{r}]$ apico-alvéolaire a disparu de la région du Centre ontarien. Nous remarquons aussi que le $[\mathrm{b}]$ dorso-vélaire est très en avance aussi dans la région de l'Est ontarien (75\%), mais que $25 \%$ des résidents pratiquent toujours le [r] apico-alvéolaire. Le Nord-Est, quant à lui, suit la tendance mais en tirant de l'arrière par rapport aux autres régions. Nous y observons $21,8 \%$ de rouleurs de $/ \mathrm{r} /$ et $32 \%$ « d'alterneurs » des /r/. Cela dit, les résultats ne permettent pas de conclure à un effet significatif de la région de la résidence officielle sur la forme de /r/ du sujet ${ }^{49}$.

\section{Usage et préférence}

Une analyse des préférences $\mathrm{du} / \mathrm{r} / \mathrm{selon}$ le $/ \mathrm{r} /$ prononcé par les sujets (figure 17) a permis de constater que ceux qui prononcent le [r] apical sont partagés entre la préférence de [r] apical (soit $43,48 \%$ ) et l'absence de préférence (soit 47,83\%). En ce qui concerne les sujets qui pratiquent le $[\mathrm{b}]$ dorsal, ils sont à leur tour divisés entre la préférence du [ $\mathrm{в}]$ dorsal (soit 57,38 \%) et l'absence de préférence (soit 42,62\%). Quant à ceux qui alternent entre les $\operatorname{deux} / \mathrm{r} /$, ils sont majoritairement campés dans la non-préférence à 76,47 \%. Il s'agit là d'un argument convaincant du fait que les locuteurs franco-ontariens ne voient pas d'un mauvais œil leurs variantes de $/ \mathrm{r} /$.

\section{Les jugements quant à l'usage}

Nous avons vu que les deux variantes $\mathrm{du} / \mathrm{r} /$ sont historiquement marquées et qu'elles ont connu des jugements aussi bien positifs que négatifs au cours de leur histoire. Mais, depuis l'établissement $\mathrm{du}[\mathrm{\zeta}]$ dorso-vélaire comme modèle normatif, pour les raisons que nous avons invoquées au préalable, les jugements négatifs sont devenus le propre du [r] apico-alvéolaire et les jugements positifs celui du [ $\mathrm{B}]$ dorso-vélaire. Il faut toutefois noter que ces jugements ont pris plus de vigueur dans les régions ou les pays majoritairement francophones ${ }^{50}$.

49. $\chi_{(8)}^{2}=13,11 ; \mathrm{p}=0,108$.

50. Denis Dumas, op. cit.; Marty Laforest, op. cit. 
Figure 17

$\mathrm{Le} / \mathrm{r} /$ préféré selon le $/ \mathrm{r} /$ prononcé (en pourcentage)

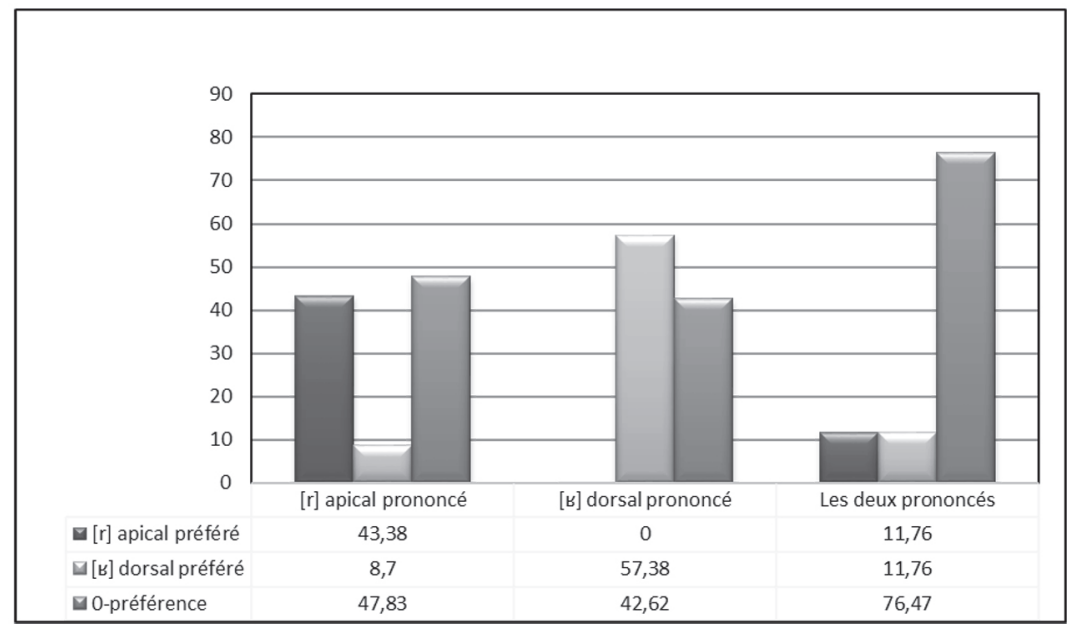

La question qui nous anime ici est de savoir si le fait de vivre dans un milieu minoritaire peut avoir un effet modérateur sur les jugements de valeur à l'endroit des deux variantes de $/ \mathrm{r} /$. Ce fut donc l'objet de notre dixième hypothèse qui stipule que, en Ontario, les jugements naturels seront plus prononcés que les jugements esthétiques quant aux usages du phonème $/ \mathrm{r} /$.

Comme nous l'avons indiqué au début de notre étude, ces jugements ont été recueillis au préalable par une préenquête où les sujets devaient écrire littéralement leurs jugements par rapport aux variantes de $/ \mathrm{r} /$. Ces jugements ont ensuite été catégorisés selon deux classes : des jugements pour les cas de préférence et d'autres pour les cas de non-préférence. Cet exercice a donné le tableau suivant : 
Tableau 1

Liste des jugements esthétiques et naturels

\begin{tabular}{|c|c|c|c|}
\hline $\begin{array}{c}\text { Jugements } \\
\text { positifs }\end{array}$ & $/ \mathrm{r} /$ préféré & $\begin{array}{c}\text { Jugements } \\
\text { négatifs }\end{array}$ & /r/ non préféré \\
\hline Esthétiques + & $\begin{array}{l}\text { Élégant, beau, sonne } \\
\text { bien, correct, sonne } \\
\text { français }\end{array}$ & Esthétiques - & $\begin{array}{l}\text { Pas beau, non } \\
\text { approprié, } \\
\text { sonne mal, } \\
\text { démodé, arriéré }\end{array}$ \\
\hline Naturels + & $\begin{array}{l}\text { Facile, exige moins } \\
\text { d'effort, familier, } \\
\text { répandu, naturel }\end{array}$ & Naturels - & $\begin{array}{l}\text { Difficile, compli- } \\
\text { qué, non naturel, } \\
\text { non appris, non } \\
\text { répandu }\end{array}$ \\
\hline
\end{tabular}

Qu'en est-il maintenant de la distribution de ces jugements à proprement parler? L'analyse des résultats (figure 18) montre que les Franco-Ontariens émettent, en général, plus de jugements naturels (33,5\% positifs et $32,67 \%$ négatifs) qu'esthétiques $(18,15 \%$ positifs et $15,38 \%$ négatifs). En outre, les résultats s'avèrent statistiquement significatifs quant à l'effet de la variante de /r/ pratiquée et la préférence pour cette même variante ${ }^{51}$. Certains diront que cela va de soi, mais cela n'est pas si évident quand plusieurs études parlent d'insécurité linguistique et d'auto-perceptions négatives. Il est vrai que la majorité des sujets pratique la variante dorsale $\mathrm{du} / \mathrm{r} /$, mais ceux qui pratiquent la variante apicale sont aussi fiers de leur variante que ne le sont les premiers. Les Franco-Ontariens jugent autant positivement que négativement la variante apicoalvéolaire en invoquant des jugements naturels, surtout quand on est détenteur de la forme dorso-vélaire, plus prestigieuse. Mais quand il s'agit d'émettre des jugements esthétiques positifs ou négatifs, la valeur baisse quasiment de $50 \%$. Cela dit, il ne faut pas oublier que plusieurs alternent entre les deux variantes de $/ \mathrm{r} /$ et, donc, cette catégorie de locuteurs ne portera probablement pas de jugements ou en portera peu.

51. $\chi_{(4)}^{2}=34,67 ; p=0,001$. 
Figure 18

Jugements pour la préférence et la non-préférence

d'une variante de $/ \mathrm{r} /$ (en pourcentage)

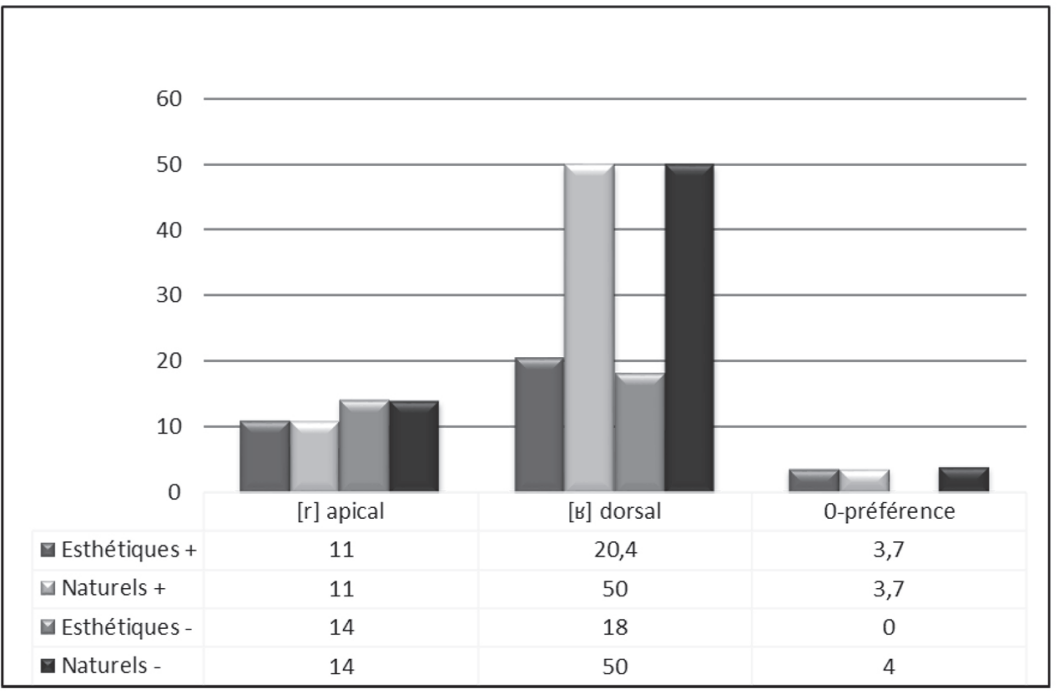

\section{CONCLUSION}

Cette recherche remet sous la loupe une vielle question pour voir si les changements sociohistoriques ont eu un impact sur la production et la perception du phonème $/ \mathrm{r}$ / en franco-ontarien. À cet effet, l'étude nous a permis de faire les constats suivants :

1. Le [r] apical perd du terrain en faveur du [в] dorsal partout en Ontario, particulièrement dans les régions du Centre et de l'Est, tant chez les hommes et les femmes, et parmi les plus jeunes, ce qui annonce un changement en cours en faveur de la normalisation du registre du phonème $/ \mathrm{r} /$ en Ontario.

2. Les jugements vis-à-vis des variantes du phonème $/ \mathrm{r} /$ connaissent un renversement, passant des jugements historiquement à dominance esthétique à des jugements à dominance naturelle et d'une montée de la tolérance envers les deux variantes par l'absence marquée de jugements.

3. Un nouveau phénomène est en train de gagner du terrain, celui de l'alternance du /r/ apical et du/r/ dorsal dans la parole d'un même locuteur en contextes extralinguistique et intralinguistique. 
C'est au niveau de ce dernier constat que nous situons la limite de notre recherche et ses perspectives. En effet, la question qui se pose pour nous dès lors est la suivante : les sons [r] et [r] sont-ils des variantes d'un même phonème / $r$ / en variation libre, ou bien des variantes contextuelles en distribution complémentaire ? D'autres recherches devraient apporter des réponses à cette question. 